\title{
Trivium
}

Revue franco-allemande de sciences humaines et sociales - Deutsch-französische Zeitschrift für Geistesund Sozialwissenschaften

$14 \mid 2013$

Le Saint-Empire à l'époque moderne

\section{Das Reichsbewusstsein des einfachen Volkes im Deutschland des 18. Jahrhunderts}

Jean-François Noël

Traducteur : Gisela Naegle

\section{CpenEdition}

Journals

Édition électronique

URL : http://journals.openedition.org/trivium/4618

DOI : $10.4000 /$ trivium.4618

ISSN : 1963-1820

Éditeur

Les éditions de la Maison des sciences de l'Homme

Référence électronique

Jean-François Noël, « Das Reichsbewusstsein des einfachen Volkes im Deutschland des

18. Jahrhunderts », Trivium [Online], 14 | 2013, online erschienen am 16 September 2013, abgerufen am 08 September 2020. URL : http://journals.openedition.org/trivium/4618 ; DOI : https://doi.org/ $10.4000 /$ trivium. 4618

Ce document a été généré automatiquement le 8 septembre 2020

\section{cc)}

Les contenus des la revue Trivium sont mis à disposition selon les termes de la Licence Creative Commons Attribution - Pas d'Utilisation Commerciale - Pas de Modification 4.0 International. 


\title{
Das Reichsbewusstsein des einfachen Volkes im Deutschland des 18. Jahrhunderts
}

\author{
Jean-François Noël \\ Traduction : Gisela Naegle
}

\section{NOTE DE L'ÉDITEUR}

Wir danken Herrn Jean-François Noël für die freundliche Genehmigung, diesen Artikel in deutscher Übersetzung zu publizieren.

Nous remercions M. Jean-François Noël de nous avoir accordé l'autorisation de traduire ce texte pour le présent numéro.

Das Überleben eines Reichspatriotismus im 18. Jahrhundert hat zwar eine sicherlich umfangreiche Literatur hervorgebracht, doch ist diese bezüglich der Quellen mit einem besonderen Handicap belastet, sobald man beabsichtigt, das Bewusstsein der Volksmassen zu erkunden. Die hochrangigeren Mitglieder der deutschen Gesellschaft die Fürsten aus politischen Gründen, die Publizisten von Berufs wegen - haben es nicht an entsprechenden Bekundungen fehlen lassen, die, ob feindselig oder nicht, aufrichtig oder nicht, bezeichnend für ihre Haltung zum späten Reich bleiben. Aber es versteht sich von selbst, dass die Bauern, Handwerker oder Händler sich weit weniger darum scherten, ihre Seelenzustände der Neugier des Historikers darzubieten.

2 Es wurden gewiss schöne, wenn nicht allzu schöne Seiten geschrieben, um zu unterstreichen, dass der sfaktischer Niedergang des Reiches seine >ideeller Verwurzelung im Volksbewusstsein nicht zum Vertrocknen gebracht hatte. Cl. Th. Perthes schrieb im letzten Jahrhundert, dass das Heilige Römische Reich, von der Kirche geweiht und mit dem Nimbus der Poesie des Mittelalters umgeben, „vom Volke mit seinem gesamten Leben verflochten " worden sei. ${ }^{1}$ Unter den Historikern der jüngeren Vergangenheit wird man hier natürlich an die von H. von Srbik inspirierte 
Historiographie denken. Selbst zu einem späten Datum, so beteuerte er, habe die Idee des Vaterlandes und des Rechts »in Millionen deutscher Seelen« nicht aufgehört, sich vom Reich zu nähren ...2

3 In Wahrheit hat Srbik die Reichsideologie in ihrer Untergangsphase vor allem in ihren »kultivierten «, literarischen und juristischen, Ausdrucksformen erfasst - nicht ohne die Stimme des Volkes ein wenig mit denen von Moser, Pütter, Goethe oder Schiller zu verwechseln ... Für eine ganze Anzahl von Historikern reduziert sich das letzte Schillern der Reichsidee nach dem Dreißigjährigen Krieg eben genau auf »ein rein literarisches Dasein [...] geknüpft an den Stand der Gebildeten, enger noch an den der Gelehrten «. ${ }^{3}$ Und auf derselben Ebene konzedieren einige ihre nur sehr marginale Repräsentativität (»vereinzelte in der Minderheit befindliche Anschauungen«). ${ }^{4}$ Auf jeden Fall wäre es vergeblich, sich hier damit aufzuhalten, historiographische Ansätze Revue passieren zu lassen, die sich, bis hin zu dem vor kurzem in englischer Sprache erschienenen Buch von J.G. Gagliardo ${ }^{5}$, in erster Linie auf die Beurteilung »höherrangiger« Zeugnisse zum Reichsbewusstsein beschränken, die nicht zu unserem Untersuchungsgegenstand gehören.

Was das breite Volk anbelangt, dessen Fall uns als einziger beschäftigen wird, so ging ein französischer »Germanist", der als Autorität galt (und immer noch gelten kann?), so weit, ernsthaft $\mathrm{zu}$ behaupten, dass sich das gesamte Leben eines Bürgers von Frankfurt oder von Mainz abspielen konnte, ohne ihnen auch nur eine einzige Gelegenheit zu geben, ihre Zugehörigkeit zu einer Körperschaft wahrzunehmen, die den Namen Deutschland trug! ${ }^{6}$ Besonders klägliche Beispiele, da es sich um die Stadt der Krönungen und den Sitz der Erzkanzlei des Reiches handelt ... Allerdings steht außer Frage, dass der normale Sterbliche im Deutschland des 18. Jahrhunderts mit einem recht beschränkten sozio-politischen Horizont lebte, der dem Dorf, der Kleinstadt oder der Herrschaft mehr Platz einräumte als dem Territorialstaat und diesem wiederum mehr Raum als einer weit entfernten und geschwächten kaiserlichen Einflusssphäre. Auf die verbleibende Frage, dennoch zu beurteilen, in welchem Ausmaß das Bewusstsein der kleinen Leute noch von einer gewissen Reichsidee geprägt war, werden wir uns im Folgenden darum bemühen, Selbstzeugnisse der Betroffenen zu zitieren. Aber um das Problem an seiner Quelle zu erfassen, ist es zunächst angebracht, bei den nicht zu vernachlässigenden materiellen Zeichen zu verweilen, die fortfuhren, den Schatten des Reiches in das Alltagsleben seiner Untertanen zu werfen.

\section{Die Umweltgegebenheiten}

5 Was den architektonischen Rahmen angeht - Denkmäler, Burgen, Rathäuser, Kirchen und Abteien, deren Namen, Legenden und Ikonographie sich so häufig mit der Reichsgeschichte verbanden -, ist es natürlich schwierig, mit Sicherheit zu sagen, bis zu welchem Grad diese Zeichen zu den ländlichen Augen und Ohren des einfachen Volkes sprechen konnten. Der deutsche Barock hatte zu jener Zeit durchaus einen Reichsstil entwickelt, der gerne aus der Reichsthematik schöpfte. ${ }^{7}$ Doch die Fresken im Kaisersaal der Würzburger Residenz, die Kaiserkronen, die die Abtei von Klosterneuburg oder die Wiener Hofburg zieren, oder auch die Darstellung Europas mit derselben Krone an der Kanzel der Abtei von Ottobeuren: all das konnte sich dem Blick oder gar der Bewunderung des großen Publikums darbieten, ohne dass dieses dabei zwangläufig die »kaiserliche« Botschaft wahrnahm. Umgekehrt wäre es aber schiere 
Arroganz des Intellektuellen, die Möglichkeit einer solchen Resonanz auszuschließen. Der einfache Mensch des Barockzeitalters war empfänglich für Formen und Farben und blieb, selbst wenn er nicht lesen konnte, oft fähig zu sehen. Im Übrigen weiß man, dass ein keineswegs gering zu schätzender Teil der Massen im 18. Jahrhundert hinreichend alphabetisiert war, so dass der Sinn kurzer Texte oder Inschriften vielen zugänglich war. Ohne es hier beweisen oder zahlenmäßig belegen zu können, wird man also zumindest vernünftigerweise für plausibel halten können, dass die plastisch-bildhafte wie schriftliche Symbolik dieses aus Jahrhunderten überkommenen Lebensrahmens schon eine gewisse Reichsvorstellung im Empfindungsvermögen des Volkes zu nähren vermochte.

Diese Möglichkeit wurde dort zur Wahrscheinlichkeit, wo eine solche Symbolik nicht nur historisch war, sondern sich auf die noch aktuelle Reichswirklichkeit bezog. Die Markierung seiner territorialen und administrativen Grenzen konnte dazu beitragen: Vom Norden, wo das Holsteiner Tor in Rendsburg den Fluss Eider als »Grenze des Römischen Reiches ${ }^{8}{ }^{8}$ anzeigte, bis hin zum tiefsten Süden, in den fernen italienischen Anhängseln dieses Reiches, wo dem einfachen Volk manchmal daran gelegen war, seine Treue herauszustellen. So suchte die ligurische Gemeinde Campo-Freddo bei Genua 1789 in Wien um die Erlaubnis nach, das kaiserliche Wappen auf ihre Tore zu malen. ${ }^{9}$

7 Eine allgemeinere Bedeutung kommt natürlich dem zu, was man den Symbolismus des Alltags nennen kann. Denken wir zunächst an das älteste ikonographische Instrument der politischen Propaganda auf der Ebene der Massen: die Münzen. Die Sorten, die im 18. Jahrhundert in Deutschland im Umlauf waren, griffen mitunter direkt auf die Reichssymbolik zurück: Adler, Krone, Bildnis und Titulatur des Kaisers etc. Der Tatbestand ließ sich sporadisch in einigen territorialen Prägestätten beobachten, besonders in reichsunmittelbaren Städten, und, in einem viel größeren Maßstab, im gesamten Verbreitungsgebiet der Münzen der Habsburger (eines in erheblichem Umfang außerösterreichischen Bereichs). Schließlich gelang es nicht einmal rein territorialen Münzen immer, sich von kaiserlichen Implikationen "frei zu machen«: teils aus technischen Gründen - ein Taler, und war er auch preußisch, musste seinen Wert als ein Reichsthaler beweisen, teils aus Gefallen an klangvollen Titulaturen - ein Kurfürst von Sachsen verschmähte es nicht, sich auf den eigenen Münzen mit seiner Eigenschaft als Reichserzmarschalls selbst zu schmeicheln..${ }^{10}$ Unterstreichen wir, dass - durchaus über das Trägermedium der Münzen hinaus - viele Fürsten und andere Territorialherren sich in ihren Rechtsakten nicht benennen konnten, ohne in den Augen ihrer Untertanen auf einige prunkvolle Würden Bezug zu nehmen, die sie an das Reich banden.

Über die Tätigkeit der kaiserlichen Notare fanden sich unzählige Dokumente nach den Regierungsjahren der Kaiser datiert. Und die Stempel, die sie trugen, transportierten mitunter eigenartige ideologische Beschwörungen, wie beispielsweise bis ins französischsprachige Bistum Lüttich: »Je recours à César, j'obéis à César, je suis protégé par l'aigle à deux têtes« (»Ich nehme meine Zuflucht zu Cäsar, ich gehorche Cäsar, ich werde vom zweiköpfigen Adler beschützt«). ${ }^{11}$

9 Die Existenz des Reiches wurde dem gewöhnlichen Sterblichen auch regelmäßig durch das Kirchengebet - ein öffentliches Gebet, das die Kirchen dem Kaiser widmeten - in Erinnerung gerufen. ${ }^{12}$ Mit einer einzigen und späten Ausnahme - man wird erraten, dass es sich um Preußen handelte, wo Friedrich II. diese "alte und übelausgedachte Gewohnheit «13 abschaffte - blieb diese überall in Gebrauch. Und als sich 1777 in den 
deutschen Teilen des Bistums Metz Priester französischer Herkunft dem entziehen wollten, mussten sie angesichts des Protestes ihrer Pfarrkinder wohl oder übel darauf verzichten. ${ }^{14}$ Zusätzlich zu dieser wöchentlichen Erinnerung waren die wichtigsten Momente jeder Regierungszeit eines Kaisers bei Gelegenheit Gegenstand von Zeremonien, die in zahlreichen volkstümlichen Liedern und Dichtungen - wie sie etwa F. W. v. Ditfurth gesammelt hat - ihre Fortsetzung erfuhren. ${ }^{15}$ So sang man zur Geburt Josephs II.: »Es bleibt beim Haus von Österreich / Der Adler von dem Teutschen Reich ... «16 Am Ende des Siebenjährigen Krieges freute man sich genauso: »Nach langem Kriegsgetöse hat nun ganz Deutschland frohen Mut / Freuet euch, ihr Deutschen alle / Vivat Kaiser Joseph ... « ${ }^{17}$ Etwas später war es die von den Mächtigen oft negativ beurteilte Reformtätigkeit Josephs, die den Enthusiasmus populärer Flugschriften mit bezeichnenden Titeln hervorrief: »Ha! Josef, Teutschlands großer Kaiser, lebe! $\aleph^{18}{ }_{[0}$ »Deutschlands Kaiser, der That auf That gehäufet! «19

Beim Tod des Kaisers war es üblich, eine Trauerzeit einzuhalten, die in vielen geistlichen Territorien bis zu 400 Tage dauern konnte, anderswo aber meistens auf eine »kleine« Kammertrauer von vier Wochen reduziert war, was dennoch ausreichte, die Öffentlichkeit mit wiederholtem Glockengeläut, Altarschmuck und Grabreden zu beeindrucken. ${ }^{20}$ Das anlässlich des Todes Josephs II. öffentlich im Regensburger Dom verlesene Gebet zeugt eindrücklich von dem gleichzeitig auf Deutschland und das Reich bezogenen Patriotismus, der solche Gedenkfeiern kennzeichnete:

»Weinet, deutsche Brüder [...], - Vater Joseph ist dahin! [...] Gott, durch den die Fürsten herrschen, uns zum Segen, uns zur Freude, gabst Du Joseph Deutschlands Thron. Du hast ihn zum Oberhaupt der Deutschen gesezt, und unter seiner Regierung unser gemeinsames Vaterland von großen Plagen verschont bleiben lassen. Siehe insonderheit mit väterlichem Erbarmen herab auf unser verwaistes Deutsches Vaterland «. ${ }^{21}$

Eine nicht zu vernachlässigende Anzahl einfacher Bürger des Reiches konnte auch und sei es nur einmal in ihrem Leben - das Privileg genießen, den Kaiser persönlich zu sehen - oder zumindest jemanden, der ihn gesehen hatte ... Wir werden im Folgenden noch auf den Fall der armen Leute zurückkommen, die ihn tatsächlich in Wien aufsuchten. Unter den anderen Gelegenheiten des Zusammentreffens hatten die Kaiserkrönungen zwar sicherlich nur einige Tausend privilegierte Zuschauer, aber hier ist die Wirkung der langen und feierlichen Züge hinzurechnen, die diese Krönungen von Wien bis nach Frankfurt in ganz Süddeutschland auslösten und die zahlreichen Dorfbewohnern die Möglichkeit gaben, den Kaiser vor ihrer Haustür vorbeiziehen zu sehen. $\mathrm{Zu}$ diesen Prozessionsdarbietungen kamen noch die Geleitzüge der Reichsinsignien hinzu, die aus Nürnberg und Aachen gebracht wurden und die zahlreiche Augenzeugen zu erreichen vermochten.

11 Unabhängig von den Krönungen kam es im 18. Jahrhundert zu relativ häufigen Reisen des Kaisers durch Deutschland, die dem Souverän jeweils Gelegenheit boten, seine Existenz und seinen Rang zu demonstrieren. Landgraf Ludwig VIII. von HessenDarmstadt dürfte seinen Untertanen einiges zu denken gegeben haben, als sie sahen, wie dieser kranke Siebzigjährige 1764 in Heusenstamm öffentlich zu Füßen des Kaisers Franz I. niederkniete ...22 Württemberg bot 1777 ebenfalls ein recht erbauliches Schauspiel. Joseph II. war gerade dabei, auf dem Weg nach Versailles das Land zu durchqueren. Herzog Karl-Eugen hatte dem Kaiser die Gastlichkeit seines Stuttgarter Palastes angeboten, aber der nüchtern-strenge Joseph hatte ihm geantwortet, er ziehe es vor, in einem Hotel abzusteigen. Karl-Eugen befahl daraufhin allen Stuttgarter 
Hoteliers, ihre Wirtshausschilder abzumontieren, und ließ vor dem herzoglichen Schloss ein übergroßes Schild mit der Aufschrift Hotel zum römischen Kaiser aufstellen. Angesichts von so viel liebenswürdiger Hartnäckigkeit gab der Kaiser schließlich nach, und Karl-Eugen ließ es sich nicht nehmen, Joseph II. in der Verkleidung eines Herbergswirts zu bedienen. ${ }^{23}$ Der öffentliche Charakter dieser Episode - und sei es auch unter dem Deckmantel eines reizenden barocken Schwanks - zeigt, dass ein Fürst dieser Zeit noch nicht zu selbstverliebt war, um im Angesicht seiner Untertanen an einer derartigen Ehrenbezeugung keinen Gefallen zu finden.

Auf eine privatere und diffusere Art fand das populäre Bild des Reiches schließlich Eingang in die familiäre, schulische und religiöse Erziehung. Der Schriftsteller Wieland schrieb, dass man ihm in seiner Kindheit »beiläufig« von seinen Pflichten gegenüber dem Kaiser erzählte..$^{24}$ Die Aussage ist nicht überzubewerten und stammt außerdem noch aus Frankfurt, einem privilegierten kaiserlichen Milieu. Aber es gibt auch Hinweise auf eine systematischere Pädagogik in Form von Katechismen und Lehrbüchern. Zitieren wir eines davon, das an kleinen Jesuitenschulen in Oberdeutschland verteilt wurde. Auf die Frage: "In wem bestehet die Glückseeligkeit des Römisch-Teutschen Reichs?«, lautete die Antwort: »In dem, daß die Glieder des Römischen Reiches mit ihrem höchsten Oberhaupt dem Römischen Kayser sich [...] ganz eng vereinigen, und [...] auf den Wohlstand des gantzen Reichs nach alt-teutscher Redlichkeit allein ihr Absehen haben .... ${ }^{25}$ Ein überaus idealisiertes Bild, das deshalb aber nicht minder die politische Vulgata zeigt, mit der damals eine ganze Reihe junger Deutscher katechisiert wurde.

Um eine erste Bilanz des bisher Gesagten zu ziehen: Festzustellen ist, dass es in Deutschland ${ }_{20}$ einschließlich seiner großen Territorien (wenn auch in einem geringeren Grad) 嫣 bis zum Ende des 18. Jahrhunderts durchaus eine Gesamtheit materieller Gegebenheiten gegeben hat, die in der einfachen Masse seiner Untertanen eine Vorstellung von der institutionellen Wirklichkeit des Reiches zu nähren vermochten.

\section{Der Beitrag der direkten Zeugnisse}

Das, was wir gerade a priori auf Annahmen gestützt haben, die aus Umweltgegebenheiten gewonnen wurden, muss nun natürlich a posteriori so weit wie möglich anhand von direkten Zeugnissen des Volksbewusstseins überprüft werden. Unter diesem Gesichtspunkt erlauben es selbst die literarischen Werke manchmal, interessante Elemente zu sammeln. So erzählte etwa der Ritter von Lang, der übrigens der kaiserlichen Dekadenz mit viel Sarkasmus gegenüberstand, in seinen berühmten Memoiren, wie eine Zigeunerin und Wahrsagerin ihm in seiner Kindheit vorhergesagt hätte, er werde eines Tages Hofrat des Kaisers. ${ }^{26}$ Die Prophezeiung erfüllte sich nicht, bleibt aber aufschlussreich. Sie zeigt, dass eine Frau niedrigster Herkunft nicht nur das Reich kannte, sondern dass sie es von der Sache her auch gut genug kannte, um den Hofrat zu benennen - und dass sie auch bei einem Dritten dessen Existenz als ausreichend bekannt voraussetzte, um ihn in ihre "Scharlatanerie « einzubeziehen; mehr noch, dass sie glaubte, diese einem Kind gegenüber einsetzen zu können ... Wer könnte schwören, dass heute unser Conseil Constitutionnel (Verfassungsrat) Eingang in die Referenzsysteme unserer Madames Soleil ${ }^{*}$ finden würde oder dass auf der anderen 
Rheinseite eine vergleichbare Ehre dem Karlsruher Bundesverfassungsgericht zuteil würde! ...

Abgesehen von solchen Anekdoten, ist es - wie bereits gesagt - relativ schwierig, an Zeugnisse des gemeinen Mannes zu gelangen. Eine substantielle Quelle gibt es jedoch in der Form der Rechtsakte der ehemaligen höchsten Gerichte des Reiches, des Wetzlarer Reichskammergerichts und des Wiener Reichshofrates, an dem der Pythia des Ritters von Lang so viel lag. Im Folgenden wird auf Beispiele zurückgegriffen, die aus der Tätigkeit dieses Rates zur Regierungszeit Josephs II. stammen.

Im Laufe der fünfundzwanzig untersuchten Jahre (1765[01790) hat allein der Reichshofrat ungefähr 10000 Streitfälle angezogen. ${ }^{27}$ Deren geographische Herkunft ist breiter gestreut, als man es gemeinhin annimmt, wenn man von der oberflächlichen Vorstellung ausgeht, die »Reichsstreitigkeiten« wären seinerzeit zur Restausstattung jener Staaten geworden, die gleichermaßen als klein, süddeutsch und katholisch galten. Hinsichtlich der Häufigkeit von Verfahren vor den Reichsgerichten nimmt in ganz Deutschland den dritten Rang nun aber gerade Mecklenburg ein, das durch seine Größe, seinen nördlichen Breitengrad und seinen massiven Protestantismus die genannten Parameter gleich dreifach zu Fall bringt. Auf sozio-politischem Gebiet betrifft ungefähr die Hälfte der untersuchten Prozesse öffentlich-rechtliche Konflikte zwischen Territorien, einschließlich der internen Streitfälle der Reichsritterschaft. Die andere Hälfte besteht aus Klagen von Privatpersonen, die entweder gegeneinander prozessierten (und nach Entscheidungen ihrer territorialen Obrigkeiten in Berufung gingen) oder aber gegen eben diese klagten. Auf dieser Ebene kann man sagen, dass abgesehen von einem gewissen Kontingent an adeligen Landsassen oder Großkaufleuten - die große Mehrheit dieser Klagen privaten Ursprungs ist (überschlagen etwa vier Fünftel der Hälfte von 10 000, das heißt ca. 4000 oder $40 \%$ ) und aus dem einfachen Milieu der Bauern, Handwerker, Krämer etc. stammt. Damit ist allerdings bei Weitem nicht die Zahl der »kleinen Leute« erfasst, die auf diese Weise mit dem Reichshofrat in Kontakt kamen: Man muss ihre aus demselben Stand stammenden Gegner hinzuzählen (was grob genommen die Zahlen bereits fast verdoppelt). Auch sind die mehrere Personen umfassenden Parteien zu berücksichtigen (die Erben von ...), die aufeinanderfolgenden Verfahrensbeteiligten, die einander in den sich oft über lange Jahre hinziehenden Prozessen ablösten, und die juristischen Personen (Dorfgemeinden, Körperschaften etc.), die oft zahlreiche physische Personen zusammenfassten. Schließlich muss zu diesem Wiener Anteil der Strom von Parteien hinzugefügt werden, die sich an das Reichskammergericht in Wetzlar wandten. Die Gesamtzahl kann mit rund 15000 Klägern aus einfachen Verhältnissen extrapoliert werden. ${ }^{28}$ Was immer man von einer solchen auf fünfundzwanzig Jahre und den Maßstab Deutschlands bezogenen Zahl halten mag, so reicht sie doch aus, um zu belegen, dass selbst seine einfachen Einwohner in der Lage waren, das Reich wahrzunehmen, und das nicht nur im Ungefähren eines fernen Gefühls mystischer Zugehörigkeit, sondern sehr wohl als eine institutionelle Realität, die sie unmittelbar betraf oder doch mittelbar betreffen konnte.

Der Stolz, mit dem eine ganze Reihe von einfachen Klägern ihre "Reichsbürgerschaft« deklamieren, entfaltet hier seinen vollen Sinn: »[Ich] als Ein des Hl. Röm. Reichs Unterthan $\aleph^{29}$, 嚂 »[Ich] verunglückte[r] Reichsbürger « ${ }^{30}$, 㡠 »[Ich] von Koßlar [Goslar] imRömischen Reich « ${ }^{31}$, 匰 »Ich bin ein Reichs-Unterthaner unter den Graf Reißen [Grafen Reuß] «32. Einige einfache Kläger verwechselten zwar ein wenig die 
Bezeichnungen der Reichsorgane, an die sie sich wandten, und verschmolzen beispielsweise den Reichshofrat und das Reichskammergericht zu einer imaginären Hofkammer. ${ }^{33}$ Aber solche Ungenauigkeiten beweisen nur, dass die Betroffenen eine eigene Vorstellung von diesen Institutionen hatten, die nicht auf die künstliche Vermittlerrolle der Rechtspraktiker zurückführbar war.

Es wird zudem deutlich, dass die Tätigkeit der Reichsgerichtsbarkeit häufig ein kollektives Echo im Volk auslöste, das über den Kreis der direkt betroffenen einzelnen Prozessparteien hinausging. Der Magistrat bzw. die katholische Stadtregierung von Dinkelsbühl beschwerte sich 1769 in Wien darüber, dass der protestantische Pastor Busch den öffentlichen Frieden dadurch störe, dass er von der Höhe seiner Kanzel seinen Pfarrkindern verkünde, der Hofrat unterstütze sie. ${ }^{34}$ Die große Breitenwirkung der kaiserlichen Entscheidungen geht auch aus dem Gnadengesuch eines Amtmanns aus Würzburg hervor, der dem Rat $1777 \mathrm{zu}$ bedenken gab, dass seine Verurteilung Gefahr laufe, das kollektive Gelächter der Biertrinker auszulösen (»Sollte meine Verurteilung vor die Ohren der renitirenden Gemeindsglieder kommen, so würde ich ohnfehlbar ein Opfer des Gespötts und ein Gegenstand ihrer Zusammenkünften auf den Bierbäncken werden «). ${ }^{35}$ Auch der Herzog von Sachsen-Hildburghausen, der 1769/1770 durch Entscheidung des Reichshofrates für zahlungsunfähig erklärt wurde, stöhnte darüber, in den Wirtshäusern laufe das Gerücht von seiner "Absetzung" um: "Der Herzog hat seinen Unterthanen nichts mehr zu befehlen ... «. ${ }^{36}$ Und selbst bis nach Reichsitalien hinein, auf das wir bereits mit einem Beispiel hingewiesen haben, kann man sehen, wie sich das Volk am Ende der Messe nach der Verkündung eines erwarteten kaiserlichen Urteilsspruchs versammelte und dabei laut »Viva, viva il nostro imperatore Giuseppe« rief [»Es lebe, es lebe unser Kaiser Joseph «]. ${ }^{37}$

Besonders aufschlussreich in Bezug auf das Reichsgefühl in den niederen Gesellschaftsschichten sind die Klagen der Parteien, die unter das Armenrecht (jus pauperum) fielen, bzw. die jener Beschwerdeführer, die üblicherweise als Supplikanten bzw. Imploranten bezeichnet werden. Ungefähr $15 \%$ der privaten Klagen gehören in diese Kategorie. Sie wurden gewöhnlicher Weise von den Parteien selbst eingereicht, die sich - ohne sich einen Anwalt oder Prokurator leisten zu können - direkt an den Rat oder sogar die Kanzlei wandten, wenn nicht an den Kaiser höchstpersönlich. Auf diese Weise trifft man auf arme Schlucker, etwa jenen Bayern, der angab, 84 Jahre alt zu $\operatorname{sein}^{38}$, und eine ganze Reihe anderer, die mitunter vom Rhein oder der Ostsee aus hunderte Kilometer zu Fuß zurückgelegt hatten in der Hoffnung, von Caesar angehört zu werden. Diese Aktenstücke zeichnen sich durch besondere Bemerkungen aus, die je nach Fall - die Bedürftigkeit der Betroffenen (causa pauper / Armensache), ihre Anwesenheit in Wien (in aula praesens, allhier anwesend) und - soweit sie sich nicht darauf beschränkten, mit einem Kreuz zu unterschreiben - den eigenhändig verfassten Charakter ihrer Klagen (selbstverfasset) unterstreichen. Die ungeformte Orthographie dieser Dokumente entsprach oft der Zusammenhanglosigkeit oder der Lächerlichkeit der vorgebrachten Beschwerden: Ein Soldat aus Mainz wurde vorstellig, um eine seit mehreren Jahren fällig gewordene Erbschaft einzufordern, wusste aber nicht zu sagen, wo und gegen wen er tätig werden sollte (»weiß nicht, wo ich mich um solches zu befragen «) ... ${ }^{39}$ Ein Bayer erklärte, wie er zum Tode und zum Verbot, mit seiner Frau zusammenzuleben, verurteilt worden war ...40 Eine Nürnbergerin appellierte an den Kaiser, weil eine Nachbarin ihren Hund beleidigt hatte..${ }^{41}$ Übrigens hatten diese Kläger meistens vollkommen vergessen, sich in erster Instanz zunächst an ihre territorialen Obrigkeiten zu wenden, wie die sächsische Untertanin, die direkt vor dem Hofrat gegen 
die Stadt Chemnitz klagte ${ }^{42}$, oder die Klageführer aus der Oberpfalz, über die das berichterstattende Hofratsmitglied feststellte: «so zeigen sie uns doch nicht im geringsten, daß sie [...] jemals beÿ der Orts Obrigkeit Klage angestellt « ${ }^{43}$ Derartige Gesuche konnten nur massenhaft abgewiesen werden, wobei die entsprechenden Formulierungen sich auf zahlreichen Akten wiederholen: "hierorts nicht statt", "anhero nicht gewachsen", "ad primam instantiam verwiesen« etc. Viele entdeckten ihren Irrtum etwas spät, wie jener Bauer aus Mainz, der zugab: »Ich als ein ungelerhrter Bauersmann verstunde nicht, daß meine Sache anhero nicht qualificiret seye. $\aleph^{44}$ Manche, die befürchteten, bei ihrer Rückkehr den Repressalien ihrer territorialen Obrigkeiten ausgesetzt zu sein, beschränkten sich darauf, sich vom Kaiser einen Geleitsbrief in Form eines Reskripts des »Rache-Verzichts« (de non vindicando recursu) ausstellen $\mathrm{zu}$ lassen ... Dennoch bleiben diese Verfahrensentgleisungen bezeichnend für eine paradoxe Tendenz, die man folgendermaßen zusammenfassen könnte: Je sozial niedriger gestellt die Parteien waren, umso eher hegten sie die Illusion, sie seien im Stande, ihre territorialen Zuständigkeiten zu übergehen und sich direkt an Caesar zu wenden!

Das Vertrauen im Volk konnte in dieser Hinsicht die allernaivste Unverschämtheit erreichen. Eine Supplikantin aus Landshut wunderte sich dem Kaiser gegenüber, dass sie noch keine Antwort auf die Briefe erhalten hat, die sie ihm, dem Vize-Kanzler und dem Hofratspräsidenten bereits vor acht Wochen geschickt hatte: Ihr Fall sei doch dringend, denn die vom bayerischen Richter erwähnten Hungerjahre trügen nicht dazu bei, dass sich ihre zehn Kinder gedulden könnten (»Die Fehljahr werden zu lang, meiner Kinder sind 10«) ...45 Ein Sattler aus Berolzheim (Brandenburg-Ansbach) verlangte vom Kaiser nicht nur ein für ihn günstiges, sondern auch eigenhändig geschriebenes Urteil, da er sich davon eine bessere Wirkung versprach. ${ }^{46}$ Ein Bürger von Windsheim glaubte, er könne für die Dauer seines Prozesses eine jährliche Rente verlangen. ${ }^{47}$ Eine Einwohnerin von Pfalz-Neuburg gab an, sich aus dem gleichen Anlass mit einem Vorschuss von hundert Dukaten von seiner kaiserlichen Meiestett zufriedenzugeben..${ }^{48}$ Einige zeigten sich zur Stützung ihres Anliegens auf rührende Weise listig: Ein Wiener Maurer argumentierte, ein glücklicher Ausgang seines Prozesses würde es ihm ermöglichen, seine Steuern besser zu bezahlen. ${ }^{49}$ Die Frau eines Maurers aus Trier erklärte sich dazu bereit, dem Kaiser das Kapital zu überlassen, das sie vor Gericht verlangte, machte jedoch zur Bedingung, dass der Hofrat ihr die Zinsen daraus zuspreche..$^{50}$ Für den Fall, dass sie ihren Fall nicht gewönne, gab sich die Betroffene damit zufrieden, einen Reisekostenzuschuss für ihre Rückkehr nach Trier zu beantragen und dem Kaiser zu versichern, dass Gott es ihm vergelten würde. ${ }^{51}$ Ein Badener, auch er ein Maurer, verlieh seinem Fall einen »historischen« Anstrich, indem er versicherte, sein Großvater hätte einst Prinz Eugen in Ungarn das Leben gerettet und diese Heldentat fände sich noch auf einem Gemälde im Wiener Belvedere-Palast dargestellt ...52

21 Abgesehen von diesen kindischen Argumenten appellierte der größte Teil der Suppliken aus dem Volk ganz einfach an die mystische Gerechtigkeit des Kaisers. Aus der Masse diesbezüglicher Zeugnisse beschränken wir uns auf die wenigen Beispiele, die im Anschluss an diese Zeilen in der kurzen Textsammlung wiedergegeben werden. Die Aufrichtigkeit ihrer Aussagen lässt sich anhand ihrer »Orthographie» und ihrer Grammatik ermessen (siehe vor allem Texte 4 bis 7 und 14 bis 16). 

Gnadenlicht, das dem in ihrem Staub seufzenden Supplikanten ein neues Leben schencken wird« (Text 2), 㥾 »den allerhöchsten Beschützer armer gekränkter Unterthanen« (Text 8, am Ende), wird ein ganz anderer Ton als in der üblichen Sprache der Schreiber erkennbar. Die Klageführer unterstrichen die Sakralität der Gerichtsbarkeit des Heiligen Römischen Reiches, wie jene Pfälzerin, die die Reise nach Wien unternommen hatte, um sich unter den Schutz der heiligen Flügel des Adlers zu begeben (»unter den geheiligten Adlersflüglen«) (Text 3). Ausdrückliche religiöse

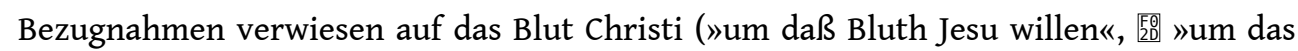
Bluth Christi«, 區 »um die fünff Wunden Jesu Christi« (Texte 4 bis 6). Die Supplik aus Sachsen-Weimar wies dem Kaiser einen unter theologischen Gesichtspunkten pikanten Platz im Rang der Heiligen Dreieinigkeit zu (»weil ich niemanden habe, der mir hilfft als dreÿ Persohnen [...] nehmlich Ihro Kaÿserliche Majestaed und Gott Vater und Gott Sohn«) (Text 4). Und die Bibelkenntnis, die ein mecklenburgischer Schulmeister zur Schau stellte, inspirierte ihn zu keinem sehr schulgerechten Deutsch (»Da ich weit von sie entfernt bin ...«) (Text 7).

Das mystisch-religiöse Element verschwand hinter dem politischen, wenn sich ein Handwerker aus Bayreuth über die calvinistischen und »souveränen« Prinzipien seines Fürsten beschwerte (Text 8), während ein ehemaliger bayerischer Hauptmann die Unterstützung feierte, die die löbliche Verfassung des deutschen Reiches den Schwachen gewähre (Text 9). Wie wir bereits haben sehen können ${ }^{53}$, ist darüberhinaus hervorzuheben, wie stark das Reichsgefühl in weiten Kreisen damals schon dem deutschen Nationalgefühl entsprach. Der Kaiser ist der Kaiser Deutschlands: »Oberhaupt des gantzen theutschen landes« (Text 8), „Deutschlands glorwürdigstes Reichs-Oberhaupt« (Text 10), »das Allerhöchste Oberhaupt des teutschen Vatterlands« (Text 12). darin zum Ausdruck, dass sie den Kaiser gern mit seinem Vornamen personalisierten: »das kan nur Joseph der Zweyte «(Text 10), 覧 »der prë̈ßwürdigste weltbekannte Eÿffer einesJoseph des 2.ten« (Text 12), "Joseph ... der allergerechteste auf Erden« (Text 13). Es handelte sich hier nicht um einen bloßen Gemeinplatz: Ganz im Gegenteil, die "normalen« Rechtstexte richteten sich immer in unpersönlicher Form an »den Kaiser«. Die namentliche Zuwendung, die die »kleinen Leute« einem Joseph II. zuteil werden ließen, legt deutlich nahe, dass das Echo seiner Reformbestrebungen bei ihnen angekommen war: »die Wäldt döhneth davon, ein müldjätiges Haubt des Römüschen Rechs Regüreth« (Text 14) ...

Auch hier kommt die ursprüngliche Authentizität dieser auf das Reich bezogenen Kultur des Volkes gerade auch in der Ungenauigkeit ihrer Informationen zum Ausdruck, so wie es bereits anhand der Terminologie der Reichsinstitutionen zu sehen war. Greifen wir hier zwei Beispiele heraus. Text 15 setzt einen thüringischen Bauern in Szene, den ein Amtmann des Grafen von Reuß wegen der verfrühten (anticibirt!) Ausübung seiner ehelichen Rechte zu einer Geldstrafe verurteilt hatte. Der Kläger verlangte, gemäß den kürzlich von Joseph II. abgemilderten Strafgesetzen bestraft zu werden. Der Amtmann hatte ein leichtes Spiel, ihm zu entgegnen, dass diese Gesetze ausschließlich in den österreichischen Erbländern angewendet würden, und dass in dieser Hinsicht 踾 so wie es ein Legist Philipps des Schönen ausgedrückt hätte - der Graf von Reuß, Heinrich XLII. (sic), Kaiser in seiner Grafschaft sei (hir wäre Heinrich der 42.te 
Kaÿser). Einmal abgesehen von der Schärfe der gräflichen Arroganz, die sich in ihr ausdrückt, unterstreicht die Anekdote, dass ein Bauer aus Thüringen durchaus gehört hatte, dass man lobend von den Reformen Josephs II. in seinen Erbländern sprach, und dass er nicht zögerte, ihren Geltungsbereich auf die Ebene des ganzen Reiches auszudehnen.

Text 16 bietet ein ähnliches Fallbeispiel für die Art, wie ein Bauer (diesmal aus Bayern) über die Wirklichkeit des Reiches zugleich informiert und in illusionärer Form desinformiert sein konnte. Das Ereignis, auf das er sich bezieht - und die genaue Angabe des Datums von 1766 erlaubt daran keinen Zweifel - geht auf ein 1766 von Joseph II. zur Reform des Reichshofrats erlassenes Dekret zurück. Es handelte sich dabei um einen Rechtsakt, der zwar sicherlich wichtig, insgesamt gesehen aber eher technischer Natur war, da er ausschließlich die interne Funktionsweise dieses Rates betraf (heute ist er im Übrigen nur noch Spezialisten bekannt). Es ist also ziemlich bemerkenswert, dass unser Bauer davon Wind bekommen hatte, und zwar so, dass er daraus ein "weldtkundig" Ereignis machte. Er übertrieb auch die Auswirkungen dieser Reform, die in Wirklichkeit überhaupt nicht den Rechtsweg für Berufungsklagen von Untertanen der Territorien an das Reich erweiterte. Der Kaiser hatte sie auch gar nicht in Bayern 㡠 und ebenso wenig anderswo 澄 »veröffentlichen« lassen (»in dem gantzen Römischen Reÿch, wie auch in gantz Beÿern Bupliciren«) ...

Die Täuschung bleibt bezeichnend für die Art, wie das Reich noch in seiner Endphase die Aufmerksamkeit seiner einfachen Untertanen auf sich lenkte. Ohne Zweifel geschah das über eine mündliche Weitergabe von Informationen, denn die Rolle der Presse, die sich in anderen Milieus als so aussagekräftig für die Vitalität des Reichspatriotismus erweist, kann hier vernachlässigt werden.

Die Geschichte des Reichspatriotismus kann sich also, wie man sieht, nicht mit einem methodischen Ansatz begnügen, der sich nur zu oft auf die obersten, politisierten und intellektualisierten Kreise der deutschen Gesellschaft beschränkt. Das Hauptziel der vorangegangenen Zeilen war, einige Ausblicke auf den zusätzlichen Gewinn zu eröffnen, den man aus Zeugnissen aus dem Volk ziehen kann. So ist deutlich geworden, dass diese die immer noch lebendigen Beziehungen veranschaulichen, die das einfache Volk mit den Institutionen des Reichs unterhielt, und das besonders auf dem Weg über Klagen vor seinen Gerichten. Hier haben wir eine Tatsache - und es gibt eine ganze Reihe weiterer, die damit übereinstimmen -, die davon abschrecken sollte, das Reichsbewusstsein des 18. Jahrhunderts auf das steingewordene Überleben eines einfachen Gefühls kollektiver Identität zu beschränken, $d$. h. auf eine bloße historische oder mystische Ideologie (»eine rein ideelle Macht«). ${ }^{54}$ Diese Versuchung zur Reduktion überlagert übrigens in eigenartiger Weise die vermeintlich übergreifenden Positionen eines Srbik, die am Anfang dieses Textes angesprochen wurden. Der »überzeitliche» Ansatz $^{55}$ seiner Schule und die von ihr ausgelöste Kritik unterscheiden sich mehr durch die (von einer Mehrheit praktizierte) »Romantisierung" bzw. den (von einer Minderheit vertretenen) »Realismus« des getroffenen Urteils als durch die Natur der Feststellung selbst. Was den inhaltlichen Kern anbelangt, glaubte Srbik selbst, das Reich als politische Größe habe schließlich damit geendet, ein "vornehmlich geistiges Dasein $\aleph^{56} \mathrm{zu}$ führen, dem er letztendlich die staatliche Qualität absprach (»Das Reich war [...] kein Staat mehr, kein organisches Wesen mit lebendiger Verfassung ()$^{57}{ }^{57}$ Mit dieser Aussage beraubte sich Srbik der Möglichkeit, die allgemeine Gültigkeit seiner Positionen dadurch abzusichern, dass er alles das mit einbezog, was sich das Reich des 
18. Jahrhunderts noch an realer, kultureller und affektiver, aber auch an organischer Substanz bewahren konnte - eine Substanz, die als solche selbst von denjenigen seiner Untertanen - der Masse der einfachen Leute - wahrgenommen wurde, die die Macht der Territorien am weitesten von ihm entfernte.

\section{Auszüge aus »Suppliken« aus dem Volk an Kaiser Joseph II. ${ }^{58}$}

6. - 1784, Örtel [JP] @ Stadt Nördlingen:

»Euer Kaÿserlichen Mayestätt werffe ich mich [...] zu Füeßen und bitte um die fünff Wunden Jesu Christi willen eine gnädigste Erhörung meiner mehr als zu großen armen und elendmühseeligen Umständen. Euer Mayestätt bitte ich fußfälligst um daß theure Blut Jesu Christi willen, mich armen Örtel nicht zu verlassen, wo Gott der allmächtige im Himel und auf Erden, über seine Mayestätt und über daß hoche Hauß Oesterreich, zeit lebenslänglich vergälder sein wollen, bis ins das spettedeste Alterthum« (DR $883 / 12)$.

\section{7. - 1787, Nehl, Schulmeister @ Herzog von Mecklemburg:}

„Da ich weit von sie entfernt bin, so habe ich erfahren, daß sie die Befehle unseres Hochgelobten Heilandes Jesu Christi nachzukommen suchen [...]. Aber über Meklen Burch muß ich leider klagen, daß es so gehet wie es im Hesekiel am 22. stehet [...]. Also wende ich mich zu Eurer Hohe Keiserliche Majesteten [...], weil sie doch Oberhaupt des gantzen theutschen landes sind« (DR, 831/6). 
»Ich bin ein Reichs Unterthaner unter den Graff Reißen (Reuß) [...], und ist mir wegen meines anticibirten Beischlafs mit meiner Ehefrau so vor der Trauung und an der Verlöbnis geschehen, $16 \mathrm{fl}$. Straff angesetzet [...]. Und da ich nun den Amtmann erinnerte, er solle so güttig sein und mich nach Kaÿser Joseph des Zweiten Rechten straffen, bekam ich aber zur Antwort, hir wäre Heinrich der 42.te Kaÿßser und wirde nicht nach Kaÿßserligen Rechten sondern nach Hochgräffligen Landes Gessetzen gerichtet. Kaÿser Joseph der Zweitte hätte wohl ein Gesetz Buch gegeben, und diese Straffen gelindert, welches aber nur in seÿnen Landen gültig und einen andern Fürsten und Herrn nichts anginge« (DR 501/3).

16. - 1771, Wölfelbauer, Bauer [JP] @ Kurfürst von Bayern:

»Es ist auch weldtkundig, daß Euer Key. Kong. Maëstäth Anno 1766 in dem gantzen Römischen Reÿch, wie auch in gantz Beÿern Bupliciren lassen, daß alle und jede, so sich 
in ihren Rechtsangelegenheiten gravirtt befinden, zu einem hochen Reuchsgericht und auch zu euer Maÿestet zu recuriren befugt seÿn sollen« (DR 1439/3).

\section{BIBLIOGRAPHIE}

Anonym (1740): Historischer Anfang [...] für die Schulen der Gesellschafft Jesu in der Ober-Teutschen Provintz, Augsburg.

Brown, K. F. E. (1787) : Über die Zurückkunft des Kaisers aus Taurien.

Büsching, A. F. (1765): Neue Erdbeschreibung, III, Bd. 3, 4. Aufl., Hamburg.

Calice, M. A. (1936): Deutscher Nationalstaat und österreichische Reichsidee, Heidelberg.

Ditfurth, F. W. von (1871 㹂1872): Historische Volkslieder der Zeit 1756 bis 1871, 2 Bde., Berlin.

Fischer, W. (1958 ) : Das Fürstentum Hohenlohe im Zeitalter der Aufklärung, Tübingen.

Gagliardo, J. G. (1980): Reich und Nation. The Holy Roman Empire as Idea and Reality, 1763熙1806, London.

Goethe, J. W. (1985): Aus meinem Leben. Dichtung und Wahrheit, 1. Teil, Buch 5, in: Sämtliche Werke nach den Epochen seines Schaffens, Bd. 16, Münchner Ausgabe, hg. von Karl Richter, München.

Häberlin, K. F. (1794): Handbuch des teutschen Staatsrechts [...], Bd. 1, Berlin.

Heer, F. (1967): Das Heilige Römische Reich, Bern / München /Wien.

Hennings, F. (1961): Und sitzet zur linken Hand. Franz Stephan von Lothringen, Gemahl der selbstregierenden Königin Maria Theresia und Römischer Kaiser, Berlin / Stuttgart / Wien.

Joachimsen, P. (1916): Vom deutschen Volk zum deutschen Staat. Eine Geschichte des deutschen Nationalbewußtseins, Berlin.

Joachimsen, P. (1983): „Vom deutschen Nationalbewußtsein«, Gesammelte Aufsätze. Beiträge [...] zum deutschen Staatsgedanken, Bd. 2., Neuauflage Aalen.

Kluckhohn, P. (1934), Die Idee des Volkes im Schrifttum der deutschen Bewegung von Moser und Herder bis Grimm, Berlin.

Koser, R. (1906): »Brandenburg-Preußen in dem Kampfe zwischen Imperialismus und reichsständischer Libertät«, Historische Zeitschrift, 96.

Lévy-Bruhl, L. (1980): L'Allemagne depuis Leibniz. Essai sur le développement de la conscience nationale en Allemagne, 1700-1848, Paris.

Madal, D. S. (1766): Vollständiges Thaler-Cabinet, Bd. 2, Königsberg.

Mommsen, W. (1952): »Zur Bedeutung des Reichsgedankens«, Historische Zeitschrift, 174.

Moser, J. J. (1767): Von dem Römischen Kayser [...], Frankfurt.

Perthes, Cl. Th. (1845): Das deutsche Staatsleben vor der Revolution, Bd. 1, Gotha.

Rothammer, W. (1781), Josef der Zweete, Germaniens großer Kaiser, Regensburg. 
Sedlmayr, H. (1977): »Die politische Bedeutung des deutschen Barock. Der Reichsstil«, Epochen und Werke, Bd. 2, Mittenwald, S. 140 医156.

Srbik, H. von (1935): Deutsche Einheit. Idee und Wirklichkeit vom Heiligen Reich bis Königgrätz, Bd. 1., München.

Srbik, H. von (1937): Österreich. Erbe und Sendung im deutschen Raum, Leipzig / Salzburg.

Vehse, E. (1853): Geschichte der deutschen Höfe seit der Reformation, Bd. 25 (Württemberg), Hamburg.

Wieland, Chr. M. (1793): »Über teutschen Patriotismus. Betrachtungen, Fragen und Zweifel«, Teutscher Merkur, Mai.

\section{NOTES}

1. Perthes (1845), S. 8 .

2. Srbik (1935), S. 129.

3. Joachimsen (1983), S. 712; siehe auch Joachimsen (1916).

4. Mommsen (1952), S. 385.

5. Gagliardo (1980).

6. Lévy-Bruhl (1980), S. 228.

7. Siehe besonders Sedlmayr (1977).

8. Büsching (1765), S. 2826.

9. Haus-, Hof- und Staatsarchiv, Wien, Reichshofrat [N.B. ohne gegenteiligen Hinweis stammen alle folgenden Archivsignaturen aus diesem Bestand], Protocollum Rerum Resolutarum 216, fol. 196, Campi Frigidi, 24.08.1789, auf eine Supplik pro consensu clementissimo imperialia insignia super binas dicti feudi portas depicta exponendi.

10. Siehe Madal (1766).

11. Siehe Judicialia Latina 484/3, Spirlet@Moles, Schriftstücke vom 07.04. und 13.12.1771, 医 443/3, (Rome) = Horion@Hessen-Rheinfels, 07.05.1781, - 443/4 Rome@Moren, 11.02.85 (verschiedene lateinische Varianten) [Zum Zeichen @ siehe Anmerkung 58; A.d.Ü.]

12. Moser (1767), S. 409; Häberlin (1794), S. 224.

13. Kabinettsorder vom 24.06.1750, zitiert von Koser (1906), S. 225.

14. Archives des Affaires étrangères [Archiv des französischen Außenministeriums], Paris, Corr. Pol. Nassau, 6, fol. 313-314, Hirtenbrief (lettre pastorale) des Bischofs von Metz zu diesem Thema, 29.06.1777.

15. Ditfurth (1871

16. Heer (1967), S. 321.

17. Ditfurth (1871), Bd. 1 (2), Lied 1, S. 1, 18.08.1765.

18. Rothammer (1781).

19. Brown (1787).

20. Moser (1767), S. 409 f., 610 f. Häberlin (1794), S. 224; siehe ein besonderes Beispiel in Fischer (1958), S. 202.

21. "Anordnung des Gottesdienstes bei dem Trauerfeste [...] über das Absterben [...] Josephs des Zweiten «, Regensburg, 1790.

22. Heer (1967), S. 332, der diese bekannte Szene beschreibt, die von Goethe in Dichtung und Wahrheit erwähnt wird. [Siehe Goethe, Aus meinem Leben. Dichtung und Wahrheit, 1. Teil, Buch 5, in: Sämtliche Werke nach den Epochen seines Schaffens, Bd. 16, Münchner Ausgabe, hg. von Karl Richter, München, 1985, S. 213 f. ; Goethe erwähnt keinen Kniefall - A.d.Ü.].

23. Vehse (1853), S. $304 \mathrm{f}$.

24. Wieland (1793), zitiert von Kluckhohn (1934). 
25. Anonyme (1740), S. 95.

26. Vehse (1853), Bd. 43 (Baierische Mediatisirte, 医 Oettingen), S. 105.

*. A.d.Ü.: Madame Soleil war eine bekannte frz. Wahrsagerin der 70er bis 90er Jahre.

27. Die statistischen Angaben dieses Abschnitts stammen aus einer laufenden Studie des Autors dieser Zeilen.

28. Rekapitulieren wir die Größenverhältnisse: ca. 4000 »einfache Kläger« in Wien, die bei Zugrundelegung einer deutlich geringeren Anzahl von Prozessgegnern der gleichen Kategorie d.h. abzüglich der Fälle, in denen diese Rolle der Territorialmacht zufällt - (nahezu) zu verdoppeln sind, was durch das Vorkommen von Mehrpersonen-Parteien wiederum nach oben zu korrigieren ist (also 8 000), erhöht schließlich noch um die bekanntermaßen etwas geringere Zahl der Prozessführer aus Wetzlar, so dass sich eine Gesamtsumme von etwa 15000 ergibt.

29. Denegata Recentiora 941/1, Preitfellner@Passau, 08.08.1774.

30. Denegata Recentiora 1389/4, Vogel@Nürnberg, 05.02.1773.

31. Denegata Recentiora 496/5, Kerl@Schneider, 24.06.1784.

32. Denegata Recentiora 501/3, Köberlein@Reuß-Plauen, 15.01.1787.

33. Denegata Recentiora 540/5, Lenard@Kurtrier, 11.11.1779.

34. Decisa 1711, Dinkelsbühl@Dinkelsbühl, 26.05.1769 (Anhang vom 17.05.1769).

35. Denegata Recentiora 938/2, Reichshoffiskal@v. Welden, 14.11.1777.

36. Obere Registratur 1296, Sachsen-Hildburghausen Schuldenwesen, 08.11.1770 (Schriftstück 132 und Anhang 168).

37. Judicialia Latina 443/7, S. Romulus (San Remo)@Genua, 09.02.1767 (Anhang).

38. Denegata Recentiora 947/13, Prag v. Monspach@Kurbayern, 09. und 23. 12. 1776.

39. Denegata Recentiora 402/7, Kleppe@x, 1775.

40. Reichshofrat Vota 7, Brunner@Kurbayern, Brief des Hofratspräsidenten Hagen, 02.12.1785.

41. Denegata Recentiora 936/1, Praunin@Hildner, 05.09.1768 (Anhang).

42. Obere Registratur 909/14, Oppelt@Chemnitz.

43. Denegata Recentiora 499/4, Kick@Kick (Votum des Berichterstatters).

44. DR 1436/14, Wald @Günzenheim, 07.11.1776.

45. Denegata Recentiora 1512/7, Ziegler(in)@Kellner, 09.01.1777.

46. Denegata Recentiora 413/6, Kühnlein@Schmidt, 26.09.1788.

47. Denegata Recentiora 540/10, Lenz@Lenz, 1779.

48. Obere Registratur 475/5, Kirchhauer@v.Lilien, 24.01.1785.

49. Obere Registratur 1558/9, Spieß@einige Bürger in Schwäbisch Gmünd, 23.09.1779.

50. Obere Registratur 1779/2, Walter@Klar, 07.08.1787.

51. Obere Registratur 1779/2, Walter@Klar, 18.12.1787.

52. Denegata Recentiora 1171/2, Schmelzel@Baden, 24.10.1774.

53. Siehe vor allem die in den Fußnoten 16, 17, 18, 19 und 21 zitierten Texte.

54. Hennings (1961), S. 285. Siehe im gleichen Sinn, Calice (1936), S. 12: »[...] die Idee [des Reiches] war im Bewußtsein des Volkes viel länger lebendig geblieben, als das Reich eine praktisch politische Bedeutung und Existenzberechtigung behalten hatte.«

55. Wir verweisen hier auf die »überzeitliche Reichsidee«, die H. v. Srbik so wichtig war und die W. Mommsen (1952) mit kritischer Ironie bedacht hat.

56. Srbik (1935), S. 129.

57. Srbik (1937), S. 129.

58. Aus: Reichshofratsakten des Haus- / Hof- und Staatsarchivs, Wien, Serie Decisa (DE), Denegata Recentiora (DR) und Obere Registratur (OR). Das Zeichen [JP] bedeutet, dass der Supplikant das Armenrecht (Jus Pauperum) in Anspruch genommen hat. Das Zeichen @ bezeichnet die öffentliche Obrigkeit, "gegen« oder "auf dem Klageweg gegen" die die Klage eingebracht wurde (im zweiten Fall wurde der Name eventueller privater Prozessgegner der Vorinstanz ausgelassen). Es versteht sich von selbst, dass die Genauigkeit der 
»orthographischen« Wiedergabe des Textes hier von jedem sic entbindet. Die Reihenfolge der Auszüge entspricht der ihrer Zitierung im oben abgedruckten Darstellungsteil.

\section{INDEX}

Mots-clés : conscience d'Empire, milieu populaire, institutions impériales, suppliques, Joseph II (empereur)

Schlüsselwörter : Reichsbewusstsein, einfaches Volk, Reichshofrat, Suppliken, Joseph II.

\section{AUTEURS}

\section{JEAN-FRANÇOIS NOËL}

Jean-François Noël ist ein französischer Historiker. Nähere Informationen finden Sie hier. 